\title{
Effect of Stress on Pattern-Dependent Oxidation of Silicon Nanostructures
}

\author{
M. Uematsu*, H. Kageshima*, K. Shiraishi** \\ * NTT Basic Research Laboratories, NTT Corporation, Atsugi 243-0198, Japan \\ uematsu@aecl.ntt.co.jp, kages@will.brl.ntt.co.jp \\ ** Institute of Physics, University of Tsukuba, Tsukuba 305-8571, Japan \\ shiraish@cm.ph.tsukuba.ac.jp
}

\begin{abstract}
Pattern-dependent oxidation (PADOX) of silicon nanostructures fabricated on siliconon-insulator (SOI) substrates is simulated. The volume expansion due to silicon oxidation is treated as a dilational strain, and the strain is applied to a transition region in which silicon is converted to oxide. In addition, the silicon oxide and transition layer are treated as viscoelastic solids. The simulation results indicate that the oxidation-induced compressive stress reduces the oxide viscosity by a few orders of magnitude and that rounded silicon shapes after oxidation are mainly caused by this reduction of oxide viscosity. In addition, we examined the relation between the oxidation-induced stress and the oxidation from below, and showed that the effect of the stress on the oxidation reaction rate affects the silicon shapes after oxidation.
\end{abstract}

\section{Introduction}

Oxidation of silicon nanostructures fabricated on SOI (silicon-on-insulator) substrates is a key process in the fabrication of silicon single-electron transistors (SETs) [1]. The oxidation is strongly affected by the initial structural size and shape, and is therefore called pattern-dependent oxidation (PADOX). The characteristic features of PADOX are mainly determined by the oxidation-induced stress and by the oxidation from below due to oxygen diffusion through the buried oxide, which pushes the silicon on the oxide upward [2]. In this paper, we present a two-dimensional simulation of PADOX and examine the effect of oxidation-induced stress on the changes of the silicon shapes after oxidation.

\section{Simulation Model}

The silicon on the oxide is pushed upward in PADOX due to the volume expansion during the oxidation. In order to take into account the upward movement, which cannot be reproduced by commercial oxidation simulators, a transition region in which silicon is converted to oxide is set up at the silicon/oxide interface (Fig. 1) [3]. The strain due to volume expansion is applied to the transition region as a dilational strain, and this expansion pushes the silicon on the oxide upward. The transition region method has been used for the simulation of LOCOS [4,5]; however, it had not been applied to PADOX. According to the expansion, strain development is solved 
and stress-strain is analyzed. The calculation flow is shown in Fig. 2. In addition, the silicon oxide and the transition layer are treated as viscoelastic solids (modeled as Maxwell body), and the stress-induced reductions of oxidation reaction, oxygen selfdiffusion in the oxide, and oxide viscosity are taken into account. The oxidation reaction rate is reduced by compressive stress normal to the interface.

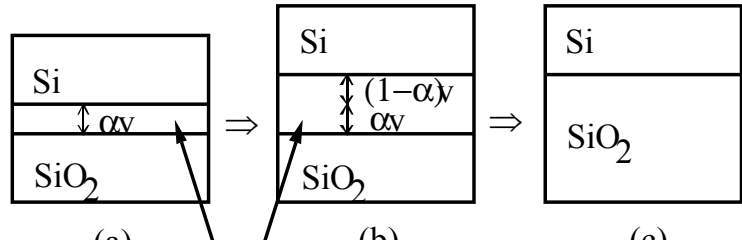

(a) transition region

(b)

(c)

Figure 1: A transition region is set up so that silicon is pushed upward. (a) Creating the transition region according to oxidant concentration obtained from oxidant concentration. (b) Volume expansion of the transition region. (c) The transition region is converted into oxide. Here, $\mathrm{v}$ is the total amount of oxidation obtained from the oxidant concentration and $\alpha$ is the volume ratio of consumed silicon.

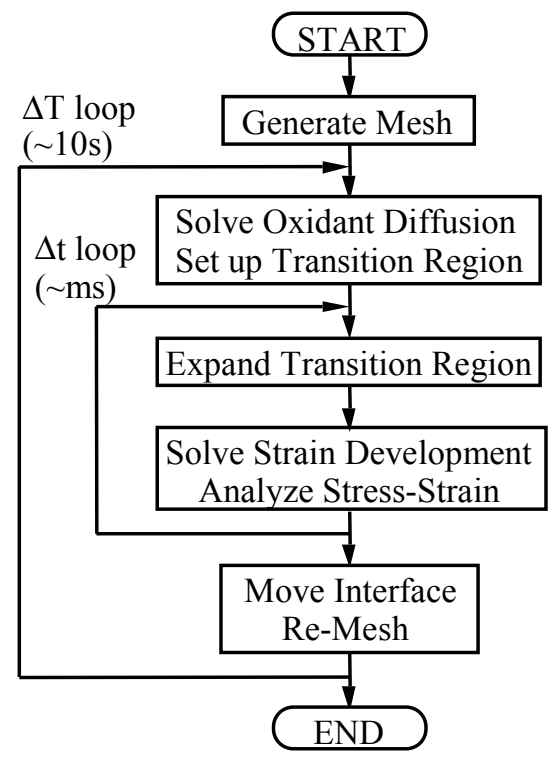

Figure 2: Calculation flow of the simulation.

\section{Simulation Results and Discussions}

The shapes of silicon and oxide after dry oxidation at $900 \sim 1100{ }^{\circ} \mathrm{C}$ were simulated and compared with the cross-sectional TEM images (Fig. 3: $1000{ }^{\circ} \mathrm{C}$ ). The initial silicon line has a width of $50 \mathrm{~nm}$ and a height of $30 \mathrm{~nm}$ with an oxide mask. The 
shapes of the silicon and oxide after oxidation were satisfactorily reproduced, which suggests that the oxidation-induced stress is properly taken into account in the simulation. The stress obtained from the simulation is compression in the silicon and in the oxide near the interface, and is of the order of $10^{10} \mathrm{dyn} / \mathrm{cm}^{2}$. The simulation results indicate that this compressive stress reduces the oxide viscosity by a few orders of magnitude and that rounded silicon shapes after oxidation (Fig. 3) are mainly caused by this reduction of oxide viscosity.

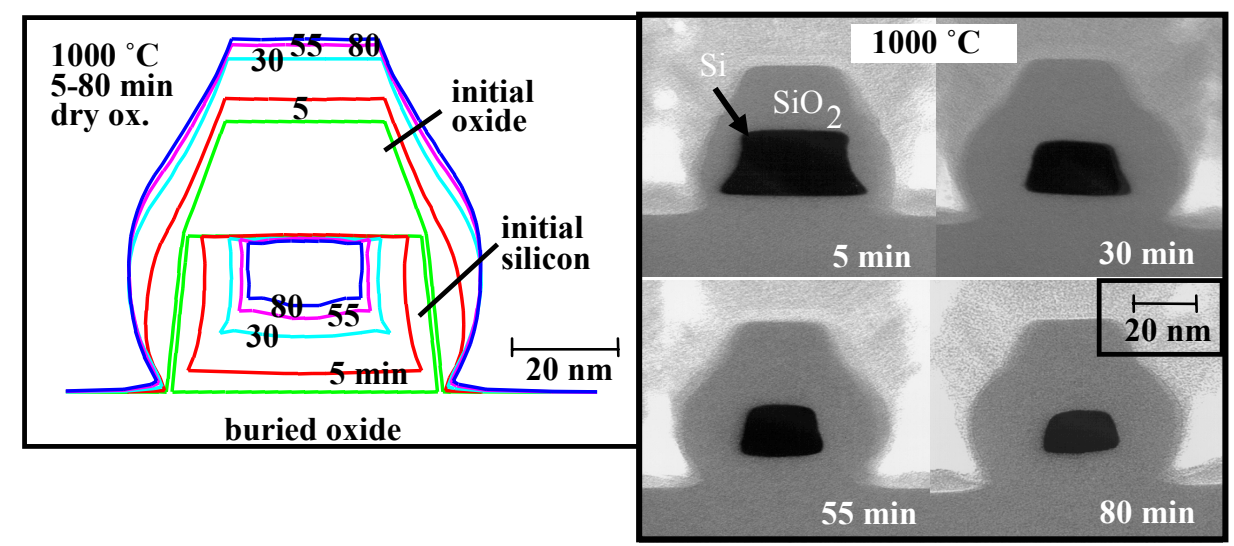

Figure 3: Simulated shapes (left) and cross-sectional TEM images (right) of silicon lines with an initial silicon width of $50 \mathrm{~nm}$ after dry oxidation at $1000^{\circ} \mathrm{C}$.

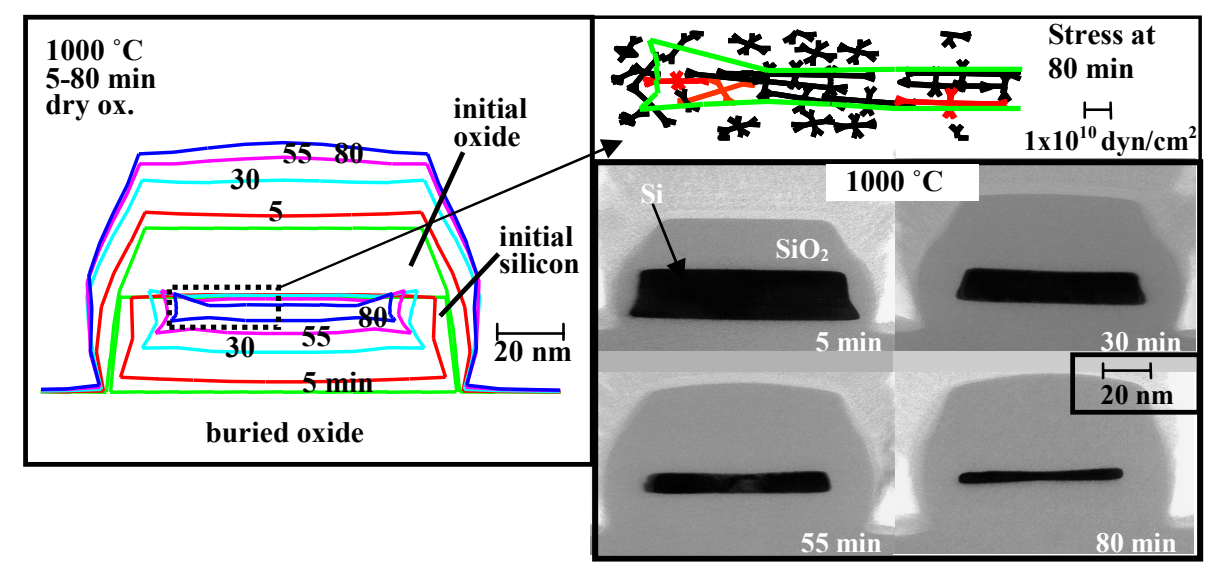

Figure 4: Simulated shapes and TEM images with an initial silicon width of $100 \mathrm{~nm}$. Calculated stress at $80 \mathrm{~min}$ is shown in the inset (upper right). 
The oxidation of the structure with an initial silicon width of $100 \mathrm{~nm}$ was also simulated (Fig. 4). The simulation well reproduces the TEM images, especially a concave shape that appears at the center of the silicon. The cause of the appearance of the concave shape is explained based on the simulation results (the inset of Fig. 4). At the left and right edges of the silicon, the silicon is surrounded by the oxide, which induces a large compressive stress both normal and parallel to the interface, leading to the reduction of the oxidation rate. At the center of the silicon, on the other hand, the normal stress is not as large as the parallel stress, because the upward movement of silicon can reduce the normal stress when the initial silicon shape is long and narrow. Therefore, the oxidation at the center is not so reduced as that at the edges, and a concave shape appears at the center of the silicon.

\section{Conclusions}

A two-dimensional simulation of PADOX on SOI substrates has been presented. The simulation results indicated that the rounded silicon shapes that appear after oxidation are mainly caused by the stress-induced reduction of oxide viscosity. In addition, we examined the relation between the oxidation-induced stress and the oxidation from below, and showed the effect of the stress on the changes of silicon shapes after oxidation.

\section{Acknowledgments}

The authors thank Mr. Y. Iriye and Mr. K. Sumino of Fuji Research Institute for the modification of the program. The authors also thank Dr. Y. Takahashi (present at Hokkaido University), Dr. M. Nagase, and Dr. A. Fujiwara of NTT for their valuable discussions.

\section{References}

[1] Y. Takahashi, M. Nagase, H. Namatsu, K. Kurihara, K. Iwadate, Y. Nakajima, S. Horiguchi, K. Murase, and M. Tabe, "Conductance oscillation of a Si single electron transistor at room temperature", IEDM Tech. Dig., pp. 938-940, 1994.

[2] M. Nagese, A. Fujiwara, K. Yamazaki, Y. Takahashi, K. Murase, and K. Kurihara, "Si nanostructures formed by pattern-dependent oxidation", Microelectron. Eng., vol. 41/42, pp. 527-530, 1998.

[3] M. Uematsu, H. Kageshima, and K. Shiraishi, Solid State Electron. "Two-Dimensional Simulation of Pattern-Dependent Oxidation of Silicon Nanostructures on Silicon-onInsulator Substrates", vol. 48, pp. 1073-1078, 2004.

[4] S. Isomae and S. Yamamoto, "A new two-dimensional silicon oxidation", IEEE Trans. CAD, vol. 6, pp. 410-416, 1987.

[5] T. Uchida, M. Fujinaga, N. Kotani, S. Kawazu, and H. Miyoshi, "Stable solution method for viscoelastic oxidation including stress-dependent viscosity", Jpn. J. Appl. Phys., vol. 35, pp. 4265-4273, 1996. 